\title{
CONVEX AND STARLIKE UNIVALENT FUNCTIONS
}

\author{
BY \\ S. D. BERNARDI
}

1. Introduction. Let $(S)$ denote the class of functions $f(z)=z+\sum_{2}^{\infty} a_{n} z^{n}$ which are regular and univalent in $|z|<1$ and which map $|z|<1$ onto domains $D(f)$. Let $(C),\left(S^{*}\right)$, and $(K)$ represent the subclasses of $(S)$ where $D(f)$ are respectively, close-to-convex, starlike with respect to the origin, and convex. It follows that $(K) \subset\left(S^{*}\right) \subset(C) \subset(S)$. We will simply say "starlike" when we mean starlike with respect to the origin, and the statement " $f(z)$ is convex" will mean that the domain $D(f)$ is convex. The abbreviations "i.o.i." and "n.s.c." have the usual meanings. Let $(P)$ denote the class of functions $p(z)$ which are reguiar and satisfy $p(0)=1$, $\operatorname{Re} p(z)>0$ for $|z|<1$. The following results, which we will use repeatedly, are well known [1]:

Let $f(z) \in(S)$. Then

$$
\begin{aligned}
& f(z) \in\left(S^{*}\right) \text { i.o.i. }\left[z f^{\prime}(z) / f(z)\right] \in(P) ; \\
& f(z) \in(K) \text { i.o.i. }\left[1+\left(z f^{\prime \prime}(z) / f^{\prime}(z)\right)\right] \in(P) ; \\
& f(z) \in(K) \text { i.o.i } \quad z f^{\prime}(z) \in\left(S^{*}\right) .
\end{aligned}
$$

If $h(z)=z+\cdots$ is regular in $|z|<1$ and $h^{\prime}(z) \in(P)$ then $h(z) \in(S)$.

In a recent paper [4] R. J. Libera established that

(1.1) if $f(z)$ is a member of $(C),\left(S^{*}\right)$ or $(K)$ then $F(z)=(2 / z) \int_{0}^{z} f(t) d t$ is also a member of the same class, respectively.

The purpose of this paper is to construct a many-parameter class of functions $F\left(c_{i} ; f\right)$ which includes the result (1.1) for special choice of parameters $c_{i}$ and, in addition, establish a subordinate relation between the image domains of certain members of $F\left(c_{i} ; f\right)$ and the image domain of the parent function $f(z)$.

2. Definitions and lemmas. The notation of $g(z) \prec f(z)$, (" $g(z)$ is subordinate to $f(z)$ "), will mean that every value taken by $g(z)$ for $|z|<1$ is also taken by $f(z)$. The convolution of two power series $f=\sum_{1}^{\infty} A_{n} z^{n}$ and $g=\sum_{1}^{\infty} B_{n} z^{n}$ is defined as the power series $f * g=\sum_{1}^{\infty} A_{n} B_{n} z^{n}$.

Definition 1. An infinite sequence $\left\{b_{n}\right\}_{1}^{\infty}$ of complex numbers is called a subordinating factor sequence (s.f.s.) if whenever $f(z) \in(K)$ we have $E(z)=f(z)$ $* \sum_{1}^{\infty} b_{n} z^{n} \prec f(z)$. This definition is due to H. S. Wilf [11] who gave n.s.c. for a sequence to be a s.f.s. It follows that the product sequence $\left\{b_{n} c_{n}\right\}_{1}^{\infty}$ of two s.f.s. $\left\{b_{n}\right\}_{1}^{\infty}$ and $\left\{c_{n}\right\}_{1}^{\infty}$ is also a s.f.s.

Received by the editors December 22, 1966 and, in revised form, September 11, 1967. 
Definition 2. An infinite sequence $\left\{d_{n}\right\}_{1}^{\infty}$ of complex numbers is said to preserve property $T$ if whenever $f=\sum_{1}^{\infty} A_{n} z^{n}$ possesses property $T$ the convolution $J(z)$ $=f * \sum_{1}^{\infty} d_{n} z^{n}$ also possesses property $T$.

Definition 3. An infinite sequence of real numbers $\left\{\mu_{n}\right\}_{0}^{\infty}$ is called a completely monotonic sequence (c.m.s.) if its elements are nonnegative and its successive differences are alternately nonpositive and nonnegative, that is,

$$
(-1)^{k} \Delta^{k} \mu_{n} \geqq 0 \quad(n, k=0,1,2, \ldots),
$$

where $\Delta^{k} \mu_{n}=\sum_{m=0}^{k}(-1)^{m} C_{k, m} \mu_{n+k-m}(k=0,1,2, \ldots)$. This definition is given by D. V. Widder $[10$, p. 108$]$ where he proves that a n.s.c. that the sequence $\left\{\mu_{n}\right\}_{0}^{\infty}$ should have the expression

$$
\mu_{n}=\int_{0}^{1} t^{n} d \alpha(t) \quad(n=0,1,2,3, \ldots)
$$

where $\alpha(t)$ is nondecreasing and bounded for $0 \leqq t \leqq 1$ is that $\left\{\mu_{n}\right\}_{0}^{\infty}$ is a c.m.s. In particular, it follows that for $c=1,2,3, \ldots$ the sequence with elements $\mu_{n}=1 /(n+c)$ is a c.m.s. and so also is the finite product sequence with elements $\mu_{n}=\Pi\left(1 /\left(n+c_{\imath}\right)\right)$, $c_{i}=1,2,3, \ldots$ It is known [2] that a c.m.s. $\left\{\mu_{n}\right\}_{0}^{\infty}$ normalized so that $\mu_{0}=1$ corresponds to a s.f.s. $\left\{b_{n}\right\}_{1}^{\infty}=\left\{\mu_{n}\right\}_{1}^{\infty}$.

Lemma A. Let $f(z) \in\left(S^{*}\right)$. Then $H(z)=z^{p-1} f(z)$ is p-valent starlike in $|z|<1$ for $p=1,2,3, \ldots$

The starlikeness of the domain $D(H)$ follows from

$$
\operatorname{Re}\left(z H^{\prime} \mid H\right)=\operatorname{Re}\left[\left(z f^{\prime} \mid f\right)+p-1\right]>p-1 \geqq 0,
$$

while the $p$-valency follows from the argument principle,

$$
\Delta(\arg H)=(p-1) \Delta(\arg z)+\Delta(\arg f)=2 \pi(p-1)+2 \pi=2 \pi p .
$$

The following lemma represents a slightly more general version of an interesting lemma proven by Libera [4].

LEMMA B. Let $f(z)$ and $g(z)$ be regular in $|z|<1, g(z)$ map $|z|<1$ onto a manysheeted starlike region, $\alpha, \beta$ real, $\operatorname{Re}\left[e^{i \beta} f^{\prime}(z) / g^{\prime}(z)\right]>\alpha$ for $|z|<1 . g(0)=f(0)=0$. Then

$$
\operatorname{Re}\left[e^{i \beta} f(z) / g(z)\right]>\alpha \text { for }|z|<1 .
$$

The method of proof is similar to that given in [4] where the lemma is proven for the case $\alpha=\beta=0$. (In this paper we use the terms $p$-valent and $p$-sheeted as being equivalent.)

Lemma C. Let $f(z) \in\left(S^{*}\right), g(z)=\int_{0}^{z} H(t) d t=\int_{0}^{z} t^{p-1} f(t) d t$. Then $g(z)$ is $(p+1)$; valent starlike for $p=1,2,3, \ldots$

The $(p+1)$-valency follows from the argument principle. To show that $g(z)$ is starlike, we note from Lemma A that $\operatorname{Re}\left(z H^{\prime} \mid H\right)>p-1$ so that

$$
\operatorname{Re}\left(z g^{\prime}\right)^{\prime} / g^{\prime}=\operatorname{Re}\left[1+z H^{\prime} \mid H\right]>p \geqq 1>0
$$


for $|z|<1$, and hence $\operatorname{Re}\left[g^{\prime} /\left(z g^{\prime}\right)^{\prime}\right]>0$. Since $z g^{\prime}=z H$ is $(p+1)$-valent starlike (Lemma A) we conclude that $\operatorname{Re}\left(g / z g^{\prime}\right)>0$ (Lemma B) or, equivalently, $\operatorname{Re}\left(z g^{\prime} / g\right)>0$ so that $g$ is starlike.

3. First generalizations. The following four theorems represent generalizations and extensions of the result (1.1). These theorems, in turn, will be the basis for further generalizations as expressed by Theorem 5 in $\S 4$.

THEOREM 1. Let $f(z)=z+\sum_{2}^{\infty} a_{n} z^{n} \in\left(S^{*}\right), c=1,2,3, \ldots$,

$$
g(z)=\sum_{n=1}^{\infty}\left(\frac{c+1}{c+n}\right) a_{n} z^{n}=(c+1) z^{-c} \int_{0}^{z} t^{c-1} f(t) d t, \quad\left(a_{1}=1\right) .
$$

Then $g(z) \in\left(S^{*}\right)$.

Corollary 1.1. For each fixed $c=1,2,3, \ldots$, the sequence $\{c /(n+c)\}_{1}^{\infty}$ is a starpreserving sequence.

Proof. Let $J(z)=\int_{0}^{z} t^{c-1} f(t) d t$. A computation gives

$$
\operatorname{Re}\left[\left(z^{c+1} g^{\prime}(z)\right)^{\prime} / J^{\prime}(z)\right]=(1+c) \operatorname{Re}\left[z f^{\prime}(z) / f(z)\right]>0 .
$$

Thus, since $J(z)$ is $(c+1)$-valent starlike (Lemma C), we may apply Lemma B and conclude that

$$
\operatorname{Re}\left[z^{c+1} g^{\prime}(z) / J(z)\right]=(1+c) \operatorname{Re}\left[z g^{\prime}(z) / g(z)\right]>0,
$$

so that $g(z)$ is starlike. The univalency of $g(z)$ then follows from the argument principle by noting that

$$
\Delta \arg (g(z))=-c \Delta(\arg z)+\Delta \arg (J(z))=-c(2 \pi)+2 \pi(c+1)=2 \pi .
$$

The corollary follows from Definition 2, where $T$ is the starlikeness property of $f(z)$, and $d_{n}=(c+1) /(c+n)$.

THEOREM 2. Let $f(z)=z+\sum_{2}^{\infty} a_{n} z^{n} \in(K), c=1,2,3, \ldots, g(z)$ the same as in Theorem 1. Then $g(z) \in(K)$, and $[c /(1+c)] g(z) \prec f(z)$.

Corollary 2.1. For each fixed $c=1,2,3, \ldots$, the sequence $\{c /(n+c)\}_{1}^{\infty}$ is a convexity-preserving subordinating factor sequence.

Proof. The function $g(z)$ may be written in the form

$$
h(z)=\left(\frac{c}{1+c}\right) g(z)=\int_{0}^{1} f(t z) d\left(t^{c}\right) .
$$

Hence $h(z)$ is the centroid of a positive mass distribution of total mass one on the curve $f(z t)$ and so lies in the convex hull of this curve. Since $f(z)$ is convex it follows that $h(z) \prec f(z)$. Also, since

$$
z g^{\prime}(z)=(1+c) z^{-c} \int_{0}^{z} t^{c-1}\left(t f^{\prime}(t)\right) d t
$$


and $z f^{\prime}(z) \in\left(S^{*}\right)$, we conclude from Theorem 1 that $z g^{\prime}(z) \in\left(S^{*}\right)$ and, therefore, $g(z) \in(K)$. The corollary follows from Definitions 1,2 .

THEOREM 3. Let $f(z)$ be close-to-convex with respect to $g(z)$,

$$
\begin{array}{r}
F(z)=(c+1) z^{-c} \int_{0}^{z} t^{c-1} f(t) d t, \quad G(z)=(c+1) z^{-c} \int_{0}^{z} t^{c-1} g(t) d t, \\
\quad c=1,2,3, \ldots .
\end{array}
$$

Then $F(z)$ is close-to-convex with respect to $G(z)$.

Corollary 3.1. For each fixed $c=1,2,3, \ldots$ the sequence $\{c /(n+c)\}_{1}^{\infty}$ is a close-to-convexity preserving sequence.

Proof. By definition of close-to-convexity [3], we are given $g(z) \in\left(S^{*}\right)$ and $\left(z f^{\prime} / g\right) \in(P)$, and we are required to prove $G(z) \in\left(S^{*}\right)$, and $\left(z F^{\prime} / G\right) \in(P)$. Theorem 1 indicates that $G(z) \in\left(S^{*}\right)$, and a computation yields

$$
\operatorname{Re}\left[\frac{\left(z^{c+1} F^{\prime}\right)^{\prime}}{\left(z^{c} G\right)^{\prime}}\right]=\operatorname{Re}\left(\frac{z^{c} f^{\prime}}{z^{c-1} g}\right)=\operatorname{Re}\left(\frac{z f^{\prime}}{g}\right)>0 .
$$

Thus, since $z^{c} G(z)$ is $(c+1)$-valent starlike (Lemma C) we may apply Lemma B and conclude that

$$
\operatorname{Re}\left(\frac{z^{c+1} F^{\prime}}{z^{c} G}\right)=\operatorname{Re}\left(\frac{z F^{\prime}}{G}\right)>0 .
$$

Corollary 3.1 follows from Definition 2 .

THEOREM 4. Let $f(z)=z+\sum_{2}^{\infty} a_{n} z^{n}$ be regular in $|z|<1, f^{\prime}(z) \in P, g(z)$ the same as in Theorem $1, c=1,2,3, \ldots$ Then $g^{\prime}(z) \in(P)$ and $g(z) \in(S)$.

This result follows immediately by noting that

$$
\operatorname{Re} g^{\prime}(z)=(c+1) \int_{0}^{1} t^{c} \operatorname{Re} f^{\prime}(t z) d t>0 .
$$

4. Further generalizations. The functions involved heretofore have the form

$$
\begin{aligned}
F(z) & =\sum_{n=1}^{\infty}\left(\frac{c+1}{n+c}\right) a_{n} z^{n}=(1+c) z^{-c} \int_{0}^{z} t^{c-1} f(t) d t \\
& =\cdot[(1+c) / c] \int_{0}^{1} f(t z) d\left(t^{c}\right)
\end{aligned}
$$

where $c=1,2,3, \ldots$ and $f(z)=z+\sum_{2}^{\infty} a_{n} z^{n}$. Theorems $1-4$ may be interpreted as properties of the sequence $\left\{b_{n}\right\}_{1}^{\infty}$, where

$$
b_{n}=\int_{0}^{1} t^{n} d \sigma(t)=\int_{0}^{1} t^{n} d\left(t^{c}\right)=c /(n+c) .
$$


In summary, the sequence (4.2) is

(a) star-preserving,

(b) convexity-preserving,

(c) close-to-convexity preserving,

(d) a subordinating factor sequence,

(e) completely monotonic.

The sequence $(c+1) /(n+c)=((c+1) / c) b_{n}$ has the same properties (a)-(c), the positive factor $(c+1) / c$ being used in (4.1) only to achieve the normalization $F^{\prime}(0)=1$.

From the preceding results and the remarks made in the Introduction, it follows that a finite product of sequences of the form (4.2) also possesses properties (a)-(e). These observations suffice as proof of Theorem 5 below. However, we supply the necessary recursive relations (4.4)-(4.11) which may be used to formalize the proof of Theorem 5 .

Let $f(z)=z+\sum_{2}^{\infty} a_{n} z^{n}$ be regular in $|z|<1$,

$$
\begin{aligned}
F_{p}(z)= & \sum_{n=1}^{\infty}\left(\frac{1+c_{1}}{n+c_{1}} \cdot \frac{1+c_{2}}{n+c_{2}} \cdots \frac{1+c_{p}}{n+c_{p}}\right) a_{n} z^{n}, \\
F_{p+1}(z)= & \sum_{n=1}^{\infty}\left(\frac{1+c_{1}}{n+c_{1}} \cdot \frac{1+c_{2}}{n+c_{2}} \cdots \frac{1+c_{p}}{n+c_{p}}\right) \cdot\left(\frac{1+c_{p+1}}{n+c_{p+1}}\right) a_{n} z^{n}, \\
& \quad\left(p, c_{i}=1,2,3, \ldots ; F_{0}(z) \equiv f(z)\right) .
\end{aligned}
$$

The $c_{i}$ may or may not be distinct. Let $g(z)=z+\sum_{2}^{\infty} d_{n} z^{n}, G_{p}(z), G_{p+1}(z)$ be similarly defined, with identical $c_{i}$ as in $F_{p}(z)$ and $F_{p+1}(z)$ but with $d_{n}$ replacing $a_{n}$. The following recursive relations now follow easily.

$$
F_{p+1}(z)=\left(1+c_{p+1}\right) z^{-c_{p+1}} \int_{0}^{z} t^{-1+c_{p+1}} F_{p}(t) d t,
$$

or, equivalently,

$$
F_{p+1}(z)=\left(1+c_{p+1}\right) \int_{0}^{1} t^{-1+c_{p+1}} F_{p}(z t) d t .
$$

Differentiate (4.5) and obtain

$$
F_{p+1}^{\prime}(z)=\left(1+c_{p+1}\right) \int_{0}^{1} t^{c_{p+1}} F_{p}^{\prime}(z t) d t .
$$

From (4.4) we have

$$
\left[z^{c_{p+1}} F_{p+1}(z)\right]^{\prime}=\left(1+c_{p+1}\right) z^{-1+c_{p+1}} F_{p}(z),
$$

and from (4.7) we obtain

$$
z F_{p+1}^{\prime}(z)+c_{p+1} F_{p+1}(z)-\left(1+c_{p+1}\right) F_{p}(z)=0 .
$$

The relation (4.6) may be written as

$$
z F_{p+1}^{\prime}(z)=\left(1+c_{p+1}\right) z^{-c_{p+1}} \int_{0}^{z} t^{-1+c_{p+1}}\left(t F_{p}^{\prime}(t)\right) d t .
$$


Differentiate (4.8), multiply by $z^{c_{p+1}}$, and obtain

$$
\left[z^{1+c_{p+1}} F_{p+1}^{\prime}(z)\right]^{\prime}=\left(1+c_{p+1}\right) z^{c_{p+1}} F_{p}^{\prime}(z) .
$$

The above recursive relations also apply, of course, to $G_{p}(z)$. From (4.7) and (4.10) it follows that

$$
\operatorname{Re} \frac{\left[z^{1+c_{p+1}} F_{p+1}^{\prime}(z)\right]^{\prime}}{\left[z^{c_{p}+1} G_{p+1}(z)\right]^{\prime}}=\operatorname{Re} \frac{z F_{p}^{\prime}(z)}{G_{p}(z)} .
$$

THEOREM 5. Let $f(z), g(z), F_{p}(z), F_{p+1}(z), G_{p}(z), G_{p+1}(z)$ be defined as in (4.3). Then for $p=1,2,3, \ldots$ we conclude that

(I) if $f(z) \in\left(S^{*}\right)$, then $F_{p}(z) \in\left(S^{*}\right)$,

(II) if $f(z) \in(K)$, then $F_{p}(z) \in(K)$, and

$$
F_{p}(z) \prec\left(\frac{1+c_{p}}{c_{p}}\right) F_{p-1}(z) \prec \prod_{i=1}^{p}\left(\frac{1+c_{i}}{c_{i}}\right) f(z),
$$

(III) if $f(z)$ is close-to-convex with respect to $g(z)$, then $F_{p}(z)$ is close-to-convex with respect to $G_{p}(z)$,

(IV) if $f^{\prime}(z) \in(P)$, then $F_{p}^{\prime}(z) \in(P)$, and $F_{p}(z) \in(S)$.

We close this section with several observations concerning the function $F_{p}(z)$ of (4.3) by considering special choices for the constants $c_{i}$ enabling us to solve explicitly for the inverse $f(z)$. Note that for the case $p=1$ relation (4.3) may be solved explicitly for the inverse to yield (writing $c_{1}=c, F_{1}(z)=F(z)$ )

$$
f(z)=\left(\frac{1}{1+c}\right) z^{1-c}\left(z^{c} F(z)\right)^{\prime} .
$$

In particular, taking $c=1$ in (4.12) gives the inverse of the function in (1.1), namely

$$
f(z)=\frac{1}{2}(z F(z))^{\prime} .
$$

In regard to the inverse relation (4.13) it was recently proven [5] that if $F(z)$ is a member of $\left(S^{*}\right),(K)$ or $(C)$ then $f(z)$ is, respectively, starlike, convex or close-toconvex for $|z|<r_{0}=1 / 2$ and the constant $1 / 2$ is the best possible in each case; if $F^{\prime}(z) \in(P)$ then $\operatorname{Re}\left(f^{\prime}(z)\right)>0$ for $|z|<\left(5^{1 / 2}-1\right) / 2$ and again this result is the best possible.

The relation (4.3) may also be solved explicitly for the inverse function $f(z)$ in the special case obtained by setting $c_{k}=k, k=1,2,3, \ldots, p$. That is, the inverse of

$$
F_{p}(z)=\sum_{n=1}^{\infty} \prod_{k=1}^{p}\left(\frac{1+k}{n+k}\right) a_{n} z^{n}
$$

may be obtained by noting that

$$
\left[z^{p} F_{p}(z)\right]^{(p)}=\sum_{n=1}^{\infty} \prod_{k=1}^{p}\left(\frac{1+k}{n+k}\right) a_{n}\left(z^{n+p}\right)^{(p)}
$$


whereupon, substituting

gives the result

$$
\left(z^{n+p}\right)^{(p)}=\prod_{k=1}^{p}(n+k) z^{n}
$$

(4.15) $\quad f(z)=\frac{1}{(1+p) !}\left[z^{p} F_{p}(z)\right]^{(p)} \quad\left(p=0,1,2,3, \ldots ; F_{0}(z)=f(z)\right)$.

The same result may be obtained from the recursive relation (4.7). For $p=1$ the relation (4.15) reduces to (4.13). In a similar way, for the same class of functions (4.14) one readily obtains

$$
z f^{\prime}(z)=\frac{1}{(p+1) !}\left[z^{p+1} F_{p}^{\prime}(z)\right]^{(p)}
$$

From (4.15) and (4.16) we conclude that the functions (4.14) satisfy

$$
\operatorname{Re} \frac{\left(z^{p+1} F_{p}^{\prime}(z)\right)^{(p)}}{\left(z^{p} G_{p}(z)\right)^{(p)}}=\operatorname{Re} \frac{z f^{\prime}(z)}{g(z)}>0
$$

for $|z|<1$ if $f(z)$ is close-to-convex with respect to $g(z)$.

5. Integral representations. In this section we find an integral representation for the general class of functions given by (4.3), through the use of the Gamma function and the theory of partial fractions. For this purpose we adopt a slightly different notation from that used in (4.3).

THEOREM 6. Let $f(z)=z+\sum_{2}^{\infty} a_{n} z^{n}$ be regular in $|z|<1 ; q, c_{i}, \gamma_{i}=1,2,3, \ldots$; $c_{i} \neq c_{j}$ for $i \neq j$;

$$
\begin{gathered}
F_{q}(z)=\sum_{n=1}^{\infty} \prod_{i=1}^{q}\left(\frac{1+c_{i}}{n+c_{i}}\right)^{\gamma_{i}} a_{n} z^{n}, \\
W_{q}(t)=\left(\prod_{k=1}^{q} c_{k^{k}}^{\gamma^{k}}\right) \sum_{i=1}^{q} t^{c_{i}-1} \sum_{j=1}^{\gamma_{i}} \frac{A_{i j}}{\left(\gamma_{i}-j\right) !}(\log 1 / t)^{\gamma_{i}-j},
\end{gathered}
$$

where the coefficients $A_{i j}$ are determined from the theory of partial fractions by the identity

$$
\prod_{i=1}^{q} \frac{1}{\left(n+c_{i}\right)^{\gamma_{i}}}=\sum_{i=1}^{q} \sum_{j=1}^{\gamma_{i}} \frac{A_{i j}}{\left(n+c_{i}\right)^{1-j+\gamma_{i}}}
$$

(If $q=1$, then $A_{11}=1$ and $A_{1 j}=0$ for $j>1$.)

Then $F_{q}(z)$ can be written in the form

where

$$
F_{q}(z)=(1 / Q) \int_{0}^{1} f(t z) W_{q}(t) d t=(1 / b(1)) \sum_{n=1}^{\infty} a_{n} b(n) z^{n}
$$

$$
\begin{aligned}
b(n) & =\int_{0}^{1} t^{n} W_{q}(t) d t=\prod_{i=1}^{q}\left(\frac{c_{i}}{n+c_{i}}\right)^{\gamma_{i}}, \\
Q & =\int_{0}^{1} t W_{q}(t) d t=b(1), \\
W_{q}(t) & \geqq 0 \quad \text { for } 0 \leqq t \leqq 1 ; \int_{0}^{1} W_{q}(t) d t=1, \\
W_{q}(1) & =0 \quad \text { for } q>1, \\
& =c_{1} \quad \text { for } q=1 \text { and } \gamma_{1}=1 .
\end{aligned}
$$


Proof. Let $r=1,2,3, \ldots$, substitute $x=-(n+c) \log t$ into the Gamma function $\Gamma(r)=\int_{0}^{\infty} x^{r-1} e^{-x} d x$ and obtain

$$
\frac{1}{(n+c)^{r}}=\frac{1}{(r-1) !} \int_{0}^{1} t^{(n+c-1)}(\log 1 / t)^{r-1} d t .
$$

In view of (5.2) and (5.9), the relation (5.3) becomes

$$
\begin{aligned}
\prod_{i=1}^{q} \frac{1}{\left(n+c_{i}\right)^{\gamma_{i}}} & =\sum_{i=1}^{q} \sum_{j=1}^{\gamma_{i}} \frac{A_{i j}}{\left(\gamma_{i}-j\right) !} \int_{0}^{1} t^{\left(n-1+c_{i}\right)}(\log 1 / t)^{\gamma_{i}-j} d t \\
& =\left(\prod_{k=1}^{q} 1 / c_{k^{k}}^{\gamma}\right) \int_{0}^{1} t^{n} W_{q}(t) d t .
\end{aligned}
$$

Substituting (5.10) into (5.1) gives

$$
F_{q}(z)=\prod_{k=1}^{q}\left(\frac{1+c_{k}}{c_{k}}\right)^{\gamma_{k}} \int_{0}^{1}\left(\sum_{n=1}^{\infty} a_{n}(t z)^{n}\right) W_{q}(t) d t=(1 / Q) \int_{0}^{1} f(t z) W_{q}(t) d t
$$

The relation (5.5) follows immediately by comparing (5.1) with (5.4) and using (5.10). Also, since the product sequence (5.5) is completely monotonic, its integral representation implies [10] that $W_{q}(t) \geqq 0$ for $0 \leqq t \leqq 1$. (It would be interesting to prove that $W_{q}(t) \geqq 0$ for $0 \leqq t \leqq 1$ directly from the theory of partial fractions, that is, using only (5.2) and (5.3).) The relation (5.8) follows directly from (5.2). This completes the proof.

The following corollary is an immediate consequence of Theorem 6 and the fact that $f \in(K)$ i.o.i. $z f^{\prime} \in\left(S^{*}\right)$.

COROLlaRY 6.1. Let $f(z), W_{q}(t)$ and $F_{q}(t)$ be the functions of Theorem 6. Then

(a) If $f(z) \in(K)$, then

$$
z F_{q}^{\prime}(z)=\frac{1}{Q}\left[W_{q}(1) f(z)-\int_{0}^{1} f(t z)\left(t W_{q}(t)\right)^{\prime} d t\right] \in\left(S^{*}\right) .
$$

(b) If $f(z) \in\left(S^{*}\right)$, then

$$
\int_{0}^{z}\left(F_{q}(t) / t\right) d t=(1 / Q) \int_{0}^{z}\left(1 / t^{2}\right)\left[\int_{0}^{t} f(x) W_{q}(x / t) d x\right] d t \in(K) .
$$

If in Theorem 6 we choose $q=\gamma_{1}=c_{1}=1$, then $W_{1}(t)=1, Q=1 / 2$ and Corollary 6.1 reduces to the results obtained in [4, Corollaries $1.1,2.1]$.

Theorem 6 enables us to generate the functions $F_{q}(z)$ by varying the choice of the weight functions $W_{q}(t)$. For instance, the choice $W_{q}(t)=1$ generates the function (1.1), while the choice $W_{q}(t)=c t^{c-1}$ generates the function (4.1). Other choices for $W_{q}(t)$ are dealt with in the following three examples.

EXAMPLE 1. In Theorem 6, take $\gamma_{i}=1$ for $i=1,2,3, \ldots, q$, write $A_{i 1}=A_{i}$ and obtain

$$
W_{q}(t)=\left(\prod_{k=1}^{q} c_{k}\right) \sum_{i=1}^{q} A_{i} t^{c_{i}-1} ; \quad Q=\prod_{i=1}^{q}\left(\frac{c_{i}}{1+c_{i}}\right)
$$


where the coefficients $A_{i}$ are determined by the identity

$$
\prod_{i=1}^{q} \frac{1}{n+c_{i}}=\sum_{i=1}^{q} \frac{A_{i}}{n+c_{i}} \quad\left(c_{i} \neq c_{j} \text { for } i \neq j\right)
$$

that is, $A_{\mathfrak{i}}=\prod_{j=1}^{q} 1 /\left(c_{j}-c_{i}\right)$, where $j \neq i$. We also obtain

$$
b(n)=\int_{0}^{1} t^{n} W_{q}(t) d t=\prod_{i=1}^{q}\left[c_{i} /\left(n+c_{i}\right)\right]
$$

so that the function $F_{q}(z)$ takes the form

$$
\begin{aligned}
F_{q}(z) & =\sum_{n=1}^{\infty} \prod_{i=1}^{q}\left(\frac{1+c_{i}}{n+c_{i}}\right) a_{n} z^{n}=(1 / Q) \int_{0}^{1} f(t z) W_{q}(t) d t \\
& =(1 / b(1)) \sum_{n=1}^{\infty} a_{n} b(n) z^{n} .
\end{aligned}
$$

In particular, for $q=1$ we have $A_{1}=1, Q=c_{1} /\left(1+c_{1}\right), W_{1}(t)=c_{1} t^{c_{1}-1}, b(n)=$ $c_{1} /\left(n+c_{1}\right)$ and

$$
F_{1}(z)=\sum_{n=1}^{\infty}\left(\frac{1+c_{1}}{n+c_{1}}\right) a_{n} z^{n}=\left(1+c_{1}\right) z^{-c_{1}} \int_{0}^{z} t^{c_{1}-1} f(t) d t,
$$

that is, the function of (4.1). Similarly, taking $q=2$ in Example 1 we obtain

$$
\begin{aligned}
W_{2}(t) & =\frac{c_{1} c_{2}}{c_{2}-c_{1}}\left(t^{c_{1}-1}-t^{c_{2}-1}\right) ; \quad Q=\frac{c_{1} c_{2}}{\left(1+c_{1}\right)\left(1+c_{2}\right)} \\
b(n) & =\int_{0}^{1} t^{n} W_{2}(t) d t=\left[c_{1} c_{2} /\left(n+c_{1}\right)\left(n+c_{2}\right)\right], \\
F_{2}(z) & =\sum_{n=1}^{\infty} \frac{\left(1+c_{1}\right)\left(1+c_{2}\right)}{\left(n+c_{1}\right)\left(n+c_{2}\right)} a_{n} z^{n} \\
& =\frac{\left(1+c_{1}\right)\left(1+c_{2}\right)}{\left(c_{2}-c_{1}\right)} z^{-1} \int_{0}^{z} f(t)\left[\left(\frac{t}{z}\right)^{c_{1}-1}-\left(\frac{t}{z}\right)^{c_{2}-1}\right] d t .
\end{aligned}
$$

EXAMPLE 2. In Theorem 6, take $q=1, \gamma_{1}=r=1,2,3, \ldots$, write $c_{1}=c=1,2,3, \ldots$ and obtain

$$
\begin{gathered}
W_{1}(t)=\left(c^{r} /(r-1) !\right) t^{c-1}[\log 1 / t]^{r-1} ; \quad Q=[c /(1+c)]^{r}, \\
b(n)=\int_{0}^{1} t^{n} W_{1}(t) d t=\frac{c^{r}}{(r-1) !} \cdot \frac{\Gamma(r)}{(n+c)^{r}}=\left(\frac{c}{n+c}\right)^{r}
\end{gathered}
$$

so that the function $F_{q}(z)=F_{1}(z)$ takes the form

$$
\begin{aligned}
F_{1}(z) & =\sum_{n=1}^{\infty}\left(\frac{1+c}{n+c}\right)^{r} a_{n} z^{n}=(1 / Q) \int_{0}^{1} f(t z) W_{1}(t) d t \\
& =\frac{(1+c)^{r}}{(r-1) !} z^{-1} \int_{0}^{z}(t / z)^{c-1} f(t)[\log z / t]^{r-1} d t
\end{aligned}
$$

In particular, for $r=1,(5.21)$ reduces to (5.15). 
EXAMPLE 3. In Theorem 6, take $c_{k}=k, \gamma_{k}=1$ for $k=1,2,3, \ldots$ and obtain

$$
\begin{gathered}
W_{q}(t)=q(1-t)^{q-1} ; \quad Q=1 /(1+q), \\
b(n)=\int_{0}^{1} t^{n} W_{q}(t) d t=\int_{0}^{1} q t^{n}(1-t)^{q-1} d t=q B(q, n+1)
\end{gathered}
$$

so that the function $F_{q}(z)$ takes the form

$$
\begin{aligned}
F_{q}(z) & =\sum_{n=1}^{\infty} \prod_{k=1}^{q}\left(\frac{1+k}{n+k}\right) a_{n} z^{n}=(1 / Q) \int_{0}^{1} f(t z) W_{q}(t) d t \\
& =q(q+1) z^{-1} \int_{0}^{z} f(t)\left(1-\frac{t}{z}\right)^{q-1} d t \\
& =q(q+1) \sum_{n=1}^{\infty} B(q, n+1) a_{n} z^{n}
\end{aligned}
$$

where $q=1,2,3, \ldots$ and $B(u, v)$ represents the Beta function. This representation is obtained by noting that

$$
q B(q, n+1)=q \int_{0}^{1} x^{n}(1-x)^{q-1} d x=\frac{q ! n !}{(q+n) !}=\prod_{k=1}^{q}\left(\frac{k}{n+k}\right) .
$$

In particular, for $q=1,(5.24)$ reduces to (1.1).

Corollary 6.2. Let $p, q, c_{i}, d_{i}=1,2,3, \ldots$,

$$
\begin{aligned}
& h_{1}(z)=\sum_{n=1}^{\infty} b_{1}(n) z^{n}=\sum_{n=1}^{\infty} \prod_{i=1}^{q}\left(\frac{c_{i}}{n+c_{i}}\right) z^{n}, \\
& h_{2}(z)=\sum_{n=1}^{\infty} b_{2}(n) z^{n}=\sum_{n=1}^{\infty} \prod_{i=1}^{q}\left(\frac{d_{i}}{n+d_{i}}\right) z^{n}, \\
& h_{3}(z)=\sum_{n=1}^{\infty} b_{3}(n) z^{n}=\sum_{n=1}^{\infty} b_{1}(n) b_{2}(n) z^{n} .
\end{aligned}
$$

Let $h_{j}(z), j=1,2,3$ map the unit disk $|z|<1$ onto the domains $D\left(h_{j}\right)$. Then

(a) the domains $D\left(h_{j}\right)$ are convex,

(b) $\operatorname{Re} h_{j}(z)>-1 / 2$ for $|z|<1, j=1,2,3$,

(c) $D\left(h_{3}\right) \subset D\left(h_{1}\right) \cap D\left(h_{2}\right)$.

Proof. The three functions $h_{j}(z)$ are each of the form $F_{p}(z)$ (but not normalized) of Theorem 5, where $f(z)=\sum a_{n} z^{n}=z /(1-z) \in(K)$ and, therefore, the domains $D\left(h_{j}\right)$ are convex. Also, by Theorem 6, we have $b_{j}(n)=\int_{0}^{1} t^{n} W_{j}(t) d t$ so that

$$
h_{j}(z)=\sum_{n=1}^{\infty} b_{j}(n) z^{n}=\int_{0}^{1} W_{j}(t) \sum_{n=1}^{\infty}(t z)^{n} d t=\int_{0}^{1}\left(\frac{t z}{1-t z}\right) W_{j}(t) \prec z /(1-z)
$$

or, equivalently, $\operatorname{Re} h_{j}(z)>-1 / 2$. Finally, since $b_{j}(n)$ is a subordinating factor sequence it follows that $h_{3}(z)$ is subordinate to both $h_{1}(z)$ and $h_{2}(z)$. We note that parts (b) and (c) also follow directly from the work of Wilf [11, Corollary 5]. 
6. Applications. For $|z|<1$ the members of the class $(K)$ satisfy certain relations among which are the following three well-known important inequalities:
(A) $\operatorname{Re} \frac{f(z)}{z}>\frac{1}{2}$,
(B) $\operatorname{Re} \frac{z f^{\prime}(z)}{f(z)}>\frac{1}{2}$,
(C) $\operatorname{Re}\left(1+\frac{z f^{\prime \prime}(z)}{f^{\prime}(z)}\right)>0$.

Thus, if $f(z) \in(K)$ the functions $F_{q}(z)$ of (5.4) also belong to $(K)$ (and, moreover, $\left.F_{q}(z) \prec(1 / Q) f(z)\right)$ and hence also satisfy the relations (A)-(C). This leads to some new inequalities (Theorems 7-11 below) satisfied by members of the class $(K)$. We will make use of relations (6.2)-(6.5) which can be directly verified with the aid of (6.2). We write $F(z)$ and $W(t)$ instead of $F_{q}(z)$ and $W_{q}(t)$, respectively.

$$
\begin{gathered}
F(z)=(1 / Q) \int_{0}^{1} f(t z) W(t) d t=(1 / z Q) \int_{0}^{z} f(u) W(u / z) d u \\
F^{(n)}(z)=(1 / Q) \int_{0}^{1} t^{n} f^{(n)}(t z) W(t) d t=\left(z^{-n} / Q\right) \int_{0}^{z} u^{n} f^{(n)}(u) W(u / z) d u \\
\left.Q\left(z^{s} F(z)\right)^{\prime} z^{1-s}=0,1,2,3, \ldots ; F_{0}(z)=f(z)\right) \\
=W\left[z F^{\prime}(z)+s F(z)\right] \\
Q z^{-s}\left[z^{1+s} F^{\prime}(z)\right]^{\prime}=Q\left[(1+s) F^{\prime}(z)+z F^{\prime \prime}(z)\right] \\
=W(1) f^{\prime}(z)+\int_{0}^{1} t f^{\prime}(t z)\left[s W(t)-(t W(t))^{\prime}\right] d t
\end{gathered}
$$

( $s$ is any real number).

Set $s=0$ in (6.4), $s=-1$ into (6.5) and obtain

$$
\begin{aligned}
& Q z F^{\prime}(z)=W(1) f(z)-\int_{0}^{1} f(t z)(t W(t))^{\prime} d t \\
& Q z F^{\prime \prime}(z)=W(1) f^{\prime}(z)-\int_{0}^{1} t f^{\prime}(t z)\left[2 W(t)+t W^{\prime}(t)\right] d t .
\end{aligned}
$$

The inequality $\operatorname{Re}(F(z) / z)>1 / 2$ takes the form

$$
\operatorname{Re}(1 / z) \int_{0}^{1} f(t z) W(t) d t>Q / 2
$$

or, equivalently,

$$
\operatorname{Re}\left(1 / z^{2}\right) \int_{0}^{z} f(t) W(t / z) d t>Q / 2
$$


The inequality $\operatorname{Re}\left(z F^{\prime}(z) / F(z)\right)>1 / 2$ becomes

$$
\operatorname{Re} \frac{W(1) f(z)-\int_{0}^{1} t f(t z) W^{\prime}(t) d t}{\int_{0}^{1} f(t z) W(t) d t}>\frac{3}{2}
$$

or, equivalently,

$$
\operatorname{Re} \frac{z W(1) f(z)-\int_{0}^{z}(t / z) f(t) W^{\prime}(t / z) d t}{\int_{0}^{z} f(t) W(t / z) d t}>\frac{3}{2} .
$$

The inequality $\operatorname{Re}\left[1+\left(z F^{\prime \prime}(z) / F^{\prime}(z)\right)\right]>0$ becomes

$$
\operatorname{Re} \frac{z W(1) f^{\prime}(z)-W^{\prime}(1) f(z)+\int_{0}^{1} f(t z)\left(t^{2} W^{\prime}(t)\right)^{\prime} d t}{W(1) f(z)-\int_{0}^{1} f(t z)(t W(t))^{\prime} d t}>1
$$

or, equivalently,

$$
\operatorname{Re} \frac{z W(1) f^{\prime}(z)-W^{\prime}(1) f(z)+(1 / z) \int_{0}^{1} f(t)\left[u^{2} w^{\prime}(u)\right]_{u=t / z}^{\prime} d t}{W(1) f(z)-(1 / z) \int_{0}^{z} f(t)\left[u W(u)^{\prime}\right]_{u=t / z} d t}>1 .
$$

Some new results for the members $f(z)$ of the class $(K)$ will now be obtained by substituting into (6.9), (6.11), (6.13) different weight functions $W(t)$ from Theorem 6 . The choice $W(t)=1, Q=1 / 2$ gives

THEOREM 7. Let $f(z) \in(K)$. Then, for $|z|<1$, we have

$$
\operatorname{Re}\left(1 / z^{2}\right) \int_{0}^{z} f(t) d t>\frac{1}{4}
$$

$$
\begin{aligned}
& \operatorname{Re} \frac{z f(z)}{\int_{0}^{z} f(t) d t}>\frac{3}{2}, \\
& \operatorname{Re} \frac{z f^{\prime}(z)}{f(z)-(1 / z) \int_{0}^{z} f(t) d t}>1 .
\end{aligned}
$$

The choice $W(t)=c t^{c-1}, Q=c /(c+1)$ gives

THEOREM 8. Let $f(z) \in(K), c=1,2,3, \ldots$ Then, for $|z|<1$, we have

$$
\operatorname{Re} z^{-(1+c)} \int_{0}^{z} t^{c-1} f(t) d t>\frac{1}{2(c+1)}
$$

$$
\begin{aligned}
& \operatorname{Re} \frac{z^{c} f(z)}{\int_{0}^{z} t^{c-1} f(t) d t}>\frac{1+2 c}{2}, \\
& \operatorname{Re} \frac{z f^{\prime}(z)}{f(z)-c z^{-c} \int_{0}^{z} t^{c-1} f(t) d t}>c .
\end{aligned}
$$

For $c=1$, Theorem 8 reduces to Theorem 7 . The choice

$$
W(t)=\left[c_{1} c_{2} /\left(c_{2}-c_{1}\right)\right]\left(t^{c_{1}-1}-t^{c_{2}-1}\right), \quad Q=c_{1} c_{2} /\left(1+c_{1}\right)\left(1+c_{2}\right)
$$

gives 
TheOREM 9. Let $f(z) \in(K), c_{1}, c_{2}=1,2,3, \ldots, c_{1} \neq c_{2}$. Then for $|z|<1$, we have

$$
\operatorname{Re}\left(1 / z^{2}\right) \int_{0}^{z} f(t)\left[(t / z)^{c_{1}-1}-(t / z)^{c_{2}-1}\right] d t>\frac{c_{2}-c_{1}}{2\left(1+c_{1}\right)\left(1+c_{2}\right)},
$$

$$
\begin{aligned}
& \operatorname{Re} \frac{\int_{0}^{z} f(t)\left[c_{2}(t / z)^{c_{2}-1}-c_{1}(t / z)^{c_{1}-1}\right] d t}{\int_{0}^{z} f(t)\left[(t / z)^{c_{1}-1}-(t / z)^{c_{2}-1}\right] d t}>\frac{1}{2}, \\
& \operatorname{Re} \frac{\left(c_{2}-c_{1}\right) z f(z)+\int_{0}^{z} f(t)\left[c_{1}^{2}(t / z)^{c_{1}-1}-c_{2}^{2}(t / z)^{c_{2}-1}\right] d t}{\int_{0}^{z} f(t)\left[c_{2}(t / z)^{c_{2}-1}-c_{1}(t / z)^{c_{1}-1}\right] d t}>0 .
\end{aligned}
$$

In particular, for $c_{1}=1$ and $c_{2}=2$ we have

$$
\operatorname{Re}\left(1 / z^{3}\right) \int_{0}^{z} f(t)(z-t) d t>\frac{1}{12}
$$

$$
\begin{aligned}
& \operatorname{Re} \frac{\int_{0}^{z} t f(t) d t}{\int_{0}^{z} f(t)(z-t) d t}>\frac{3}{2} \\
& \operatorname{Re} \frac{z^{2} f(z)-2 \int_{0}^{z} t f(t) d t}{\int_{0}^{z} f(t)(2 t-z) d t}>1 .
\end{aligned}
$$

For the choice $W(t)=\left(c^{r} /(r-1) !\right) t^{c-1}(\log 1 / t)^{r-1}$, it follows that $Q=(c /(1+c))^{r}$. In particular, for $r=1$ we obtain $W(1)=c, W^{\prime}(1)=c(c-1)$; for $r=2$ we obtain $W(1)=0, W^{\prime}(1)=-c^{2}$; for $r>2$ we obtain $W(1)=0, W^{\prime}(1)=0$. We then have

THEOREM 10. Let $f(z) \in(K), c, r=1,2,3, \ldots$ Then for $|z|<1$ it follows that

$$
\operatorname{Re} z^{-(1+c)} \int_{0}^{z} f(t) t^{c-1}(\log z / t)^{r-1} d t>(r-1) ! / 2(1+c)^{r} .
$$

In particular, for $r=c=1$ this reduces to part (A) of Theorem 7 .

(B) $\quad \operatorname{Re} \frac{\left((r-1) ! / c^{r}\right) W(1) z^{c} f(z)+(r-1) \int_{0}^{z} f(t) t^{c-1}(\log z / t)^{r-2} d t}{\int_{0}^{z} f(t) t^{c-1}(\log z / t)^{r-1} d t}>\frac{1+2 c}{2}$.

In particular, for $r=1$, this reduces to part (B) of Theorem 7; for $r>1$ it reduces to for $r=2$ it reduces to

$$
\operatorname{Re} \frac{\int_{0}^{z} f(t) t^{c-1}(\log z / t)^{r-2} d t}{\int_{0}^{z} f(t) t^{c-1}(\log z / t)^{r-1} d t}>\frac{1+2 c}{2(r-1)}
$$

$$
\operatorname{Re} \frac{\int_{0}^{z} f(t) t^{c-1} d t}{\int_{0}^{z} f(t) t^{c-1}(\log z / t) d t}>\frac{1+2 c}{2}
$$

for $r=2$ and $c=1$ it reduces to

$$
\operatorname{Re} \frac{\int_{0}^{z} f(t) d t}{\int_{0}^{z} f(t)(\log z / t) d t}>\frac{3}{2}
$$

$$
\left[W(1) z^{c+1} f^{\prime}(z)-W^{\prime}(1) z^{c} f(z)\right](r-1) !
$$

(C) $\operatorname{Re} \frac{+c^{r} \int_{0}^{z} t^{c-1} f(t)(\log z / t)^{r-3} \cdot(L+M) d t}{W(1) z^{c} f(z)(r-1) !+c^{r} \int_{0}^{z} f(t) t^{c-1}(\log z / t)^{r-2} \cdot N d t}>1$ where

$$
\begin{gathered}
L=(r-1)[r-2-(2 c-1) \log z / t], \\
M=c(c-1)(\log z / t)^{2} ; \quad N=r-1-c(\log z / t) .
\end{gathered}
$$


For $r=1$ this reduces to (C) of Theorem 8. For $r=2$ it reduces to

For $r>2$ it reduces to

$$
\operatorname{Re} \frac{z^{c} f(z)-c \int_{0}^{z} t^{c-1} f(t) d t}{\int_{0}^{z} f(t) t^{c-1}[1-c \log z / t] d t}>c .
$$

$$
\operatorname{Re} \frac{\int_{0}^{z} t^{c-1} f(t)(\log z / t)^{r-3} \cdot[(r-2)-c(\log z / t)] d t}{\int_{0}^{z} t^{c-1} f(t)(\log z / t)^{r-2} \cdot[(r-1)-c(\log z / t)] d t}>\frac{c}{r-1} .
$$

Next, choose $W(t)=q(1-t)^{q-1}$ so that $Q=1 /(1+q)$. In particular, for $q=1$ we obtain $W(1)=1, W^{\prime}(1)=0$; for $q=2$ we obtain $W(1)=0, W^{\prime}(1)=-2$; for $q>2$ we obtain $W(1)=W^{\prime}(1)=0$. We then have

THEOREM 11. Let $f(z) \in(K), q=1,2,3, \ldots$ Then for $|z|<1$ it follows that

$$
\operatorname{Re} z^{-(1+q)} \int_{0}^{z} f(t)(z-t)^{q-1} d t>1 / 2 q(1+q) \text {. }
$$

In particular, for $q=1$ this reduces to part (A) of Theorem 7 .

$$
\operatorname{Re} \frac{W(1) z^{q} f(z)+q(q-1) \int_{0}^{z} t f(t)(z-t)^{q-2} d t}{\int_{0}^{z} f(t)(z-t)^{q-1} d t}>\frac{3 q}{2} .
$$

In particular, for $q=1$ this reduces to part (B) of Theorem 7 ; for $q \geqq 2$ it reduces to

$$
\operatorname{Re} \frac{\int_{0}^{z} t f(t)(z-t)^{q-2} d t}{\int_{0}^{z} f(t)(z-t)^{q-1} d t}>\frac{3}{2(q-1)}
$$

and for $q=2$ it reduces to part $\left(\mathrm{B}_{1}\right)$ of Theorem 9 .

$$
\text { (C) } \operatorname{Re} \frac{W(1) z^{1+q} f^{\prime}(z)-W^{\prime}(1) z^{q} f(z)+q(q-1) \int_{0}^{z} t f(t)(z-t)^{q-3} \cdot(q t-2 z) d t}{W(1) z^{q} f(z)+q \int_{0}^{z} f(t)(z-t)^{q-2} \cdot(q t-z) d t}>1 \text {. }
$$

In particular, for $q=1$ this reduces to part (C) of Theorem 7 ; for $q=2$ it reduces to part $\left(\mathrm{C}_{1}\right)$ of Theorem 9 ; for $q>2$ it reduces to

$$
\operatorname{Re} \frac{\int_{0}^{z} t f(t)(z-t)^{q-3} \cdot(q t-2 z) d t}{\int_{0}^{z} f(t)(z-t)^{q-2} \cdot(q t-z) d t}>\frac{1}{q-1} .
$$

7. Recursive relations satisfied by the weight function. Recursive relations for the weight function $W_{p}(t)$ similar to those derived in $\$ 4$ for $F_{p}(t)$ will now be obtained. Also some identities satisfied by the subordinating sequence $b(n)$ will be given. The relations should be useful for the investigation of the problems and conjectures of $\S 8$. As in (4.3) let $p, c_{i}=1,2,3, \ldots, f(z)=z+\sum_{2}^{\infty} a_{n} z^{n}$ and write

$$
\begin{aligned}
F_{p}(z) & =\sum_{n=1}^{\infty} \prod_{i=1}^{p}\left(\frac{1+c_{i}}{n+c_{i}}\right) a_{n} z^{n}=\frac{1}{b_{p}(1)} \sum_{n=1}^{\infty} b_{p}(n) a_{n} z^{n} \\
& =\left(1 / Q_{p}\right) \int_{0}^{1} f(t z) W_{p}(t) d t, \\
F_{p+1}(z) & =\sum_{n=1}^{\infty} \prod_{i=1}^{p}\left(\frac{1+c_{i}}{n+c_{i}}\right) \cdot\left(\frac{1+c_{p+1}}{n+c_{p+1}}\right) a_{n} z^{n} \\
& =\frac{1}{b_{p+1}(1)} \sum_{n=1}^{\infty} b_{p+1}(n) a_{n} z^{n}=\left(1 / Q_{p+1}\right) \int_{0}^{1} f(t z) W_{p+1}(t) d t,
\end{aligned}
$$


where the $c_{i}$ may or may not be distinct, and $W_{r}(t)$ is as in (5.2) (with the appropriate change so that $\left.p=\sum_{i=1}^{q} \gamma_{i}\right)$. It follows that

$$
Q_{p+1}=\left(\frac{c_{p+1}}{1+c_{p+1}}\right) Q_{p} ; \quad W_{p+1}(1)=0 .
$$

From (4.8) we have the recursive relation

$$
z F_{p+1}^{\prime}(z)+c_{p+1} F_{p+1}(z)=\left(1+c_{p+1}\right) F_{p}(z) .
$$

Substitute into (7.4) the integral representations for $F_{p+1}(z), F_{p}(z), z F_{p+1}^{\prime}$ from (7.2), (7.1), (6.6) respectively. Then apply (7.3) and deduce the recursive relation

$$
t W_{p+1}^{\prime}(t)+\left(1-c_{p+1}\right) W_{p+1}(t)+c_{p+1} W_{p}(t)=0 \quad(p=1,2,3, \ldots) .
$$

Solve the differential equation (7.5) for $W_{p+1}(t)$ and obtain

$$
W_{p+1}(t)=c_{p+1} t^{-1+c_{p+1}}\left[K-\int t^{-c_{p+1}} W_{p}(t) d t\right] .
$$

To evaluate the constant of integration $K$, apply the condition $W_{p+1}(1)=0$ and obtain

$$
W_{p+1}(t)=c_{p+1} t^{-1+c_{p+1}} \int_{t}^{1} t^{-c_{p+1}} W_{p}(t) d t
$$

A comparison of (7.1) and (7.2) yields

$$
\left(n+c_{p+1}\right) b_{p+1}(n)=c_{p+1} b_{p}(n) \quad(n, p=1,2,3, \ldots) .
$$

Substitute into (7.7) the integral representations for $b_{p}(n)$ and $b_{p+1}(n)$ given by (5.5) and obtain the zero moment

$$
\int_{0}^{1} t^{n}\left[\left(n+c_{p+1}\right) W_{p+1}(t)-c_{p+1} W_{p}(t)\right] d t=0 .
$$

Again using (5.5) the following moment relations are directly verifiable.

$$
\begin{gathered}
b_{r}(n) b_{s}(n)=\int_{0}^{1} t^{n}\left[2 b_{s}(n) W_{r}(t)-b_{r}(n) W_{s}(t)\right] d t, \\
\int_{0}^{1} t^{n}\left[b_{s}(n) W_{r}(t)-b_{r}(n) W_{s}(t)\right] d t=0, \\
b_{r}^{k}(n)=\int_{0}^{1} t^{n} b_{r}^{k-1}(n) W_{r}(t) d t \quad(p, k, r, s=1,2,3, \ldots) .
\end{gathered}
$$

We conclude this section by applying the relation (7.6) to two examples.

EXAMPLE 1a. In (7.1) and (7.2) let $c_{i} \neq c_{j}$ for $i \neq j$. Then from (5.12) we have

$$
\begin{aligned}
W_{p}(t) & =\left(\prod_{k=1}^{p} c_{k}\right) \sum_{i=1}^{p} A_{i} t^{c_{i}-1}, \\
W_{p+1}(t) & =\left(\prod_{k=1}^{p} c_{k}\right) c_{p+1} \sum_{i=1}^{p+1} B_{i} t^{c_{i}-1},
\end{aligned}
$$

where $A_{i}$ and $B_{i}$ are defined by

$$
\prod_{i=1}^{p}\left(\frac{1}{n+c_{i}}\right)=\sum_{i=1}^{p} \frac{A_{i}}{n+c_{i}} ; \quad \prod_{i=1}^{p+1}\left(\frac{1}{n+c_{i}}\right)=\sum_{i=1}^{p+1} \frac{B_{i}}{n+c_{i}} .
$$


By the theory of partial fractions the coefficients $A_{i}$ and $B_{i}$ are related by

$$
B_{i}=\frac{A_{i}}{c_{p+1}-c_{i}} \quad(i=1,2,3, \ldots, p) ; \quad B_{p+1}=\prod_{j=1}^{p}\left(\frac{1}{c_{j}-c_{p+1}}\right)
$$

We then obtain

$$
\begin{aligned}
& K=\int_{t=1} t^{-c_{p+1}} W_{p}(t) d t=\left(\prod_{k=1}^{p} c_{k}\right) \int_{t=1} t^{-c_{p+1}} \sum_{i=1}^{p} A_{i} t^{c_{i}-1} d t \\
& =\left(\prod_{k=1}^{p} c_{k}\right) \sum_{i=1}^{p} \frac{A_{i}}{c_{i}-c_{p+1}}=\left(\prod_{k=1}^{p} c_{k}\right) \sum_{i=1}^{p}\left(-B_{i}\right) .
\end{aligned}
$$

Since $\sum_{i=1}^{p+1} B_{i}=0$, we have

$$
\sum_{i=1}^{p} B_{i}=-B_{p+1}=-\prod_{j=1}^{p}\left(1 /\left(c_{j}-c_{p+1}\right)\right)
$$

so that $K=\prod_{j=1}^{p}\left(c_{j} /\left(c_{j}-c_{p+1}\right)\right)$, and therefore (7.6) becomes

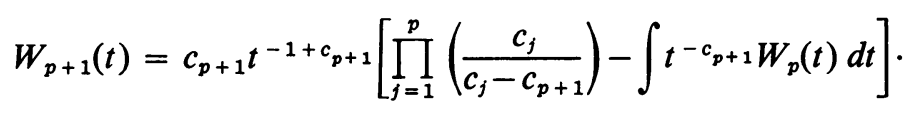

EXAMPLE 2a. In (7.1) and (7.2) let $c_{i}=c$ for $i=1,2,3, \ldots, p$ and $c_{p+1} \neq c$. Then from (5.19) we have

$$
W_{p}(t)=\frac{c^{p}}{(p-1) !} t^{c-1}(\log 1 / t)^{p-1}
$$

and so we obtain

$$
\begin{aligned}
K & =\int_{t=1} t^{-c_{p+1}} W_{p}(t) d t=\frac{c^{p}}{(p-1) !} \int_{t=1} t^{c-1-c_{p+1}(\log 1 / t)^{p-1}} d t \\
& =\left(c /\left(c-c_{p+1}\right)\right)^{p}
\end{aligned}
$$

where we have integrated by parts $(p-1)$ times. Therefore, (7.6) becomes

$$
W_{p+1}(t)=c_{p+1} t^{-1+c_{p+1}}\left[\left(\frac{c}{c-c_{p+1}}\right)^{p}-\int t^{-c_{p+1}} W_{p}(t) d t\right]
$$

Note that for $p=1,(7.15)$ and (7.17) are identical (if we write $c_{1}=c$ ).

8. Some open questions and conjectures. Let $f(z)=z+\sum_{2}^{\infty} a_{n} z^{n}$ be regular and univalent for $|z|<1$. Pólya and Schoenberg [6] have proven Theorem A and also the necessity part of Theorem B below, while Robertson [7] has proven the sufficiency part of Theorem B. 
TheOREM A. $f(z) \in(K)$ if and only if the de la Vallée Poussin means of $f(z)$, namely the polynomials

$$
\begin{aligned}
V_{n}(z ; f)= & \left(\frac{1}{C_{2 n, n}}\right) \sum_{v=1}^{n} C_{2 n, n+v} a_{v} z^{v} \\
= & \frac{n}{n+1} z+\frac{n(n-1)}{(n+1)(n+2)} a_{2} z^{2}+\cdots \\
& +\frac{n(n-1) \cdots(n-k+1)}{(n+1)(n+2) \cdots(n+k)} a_{k} z^{k}+\cdots \\
& +\frac{n(n-1) \cdots 1}{(n+1)(n+2) \cdots(2 n)} a_{n} z^{n}
\end{aligned}
$$

are univalent and convex in $|z|<1$ for $n=1,2,3, \ldots$

THEOREM B. $f(z) \in(K)$ if and only if $V_{n}(z ; f) \prec f(z)$ for $n=1,2,3, \ldots,|z|<1$.

Thus if $f(z) \in(K)$ then the sequence $\left\{b_{n}\right\}_{1}^{\infty}$ with elements

$$
\begin{aligned}
b_{n} & =\frac{k(k-1) \cdots(k-n+1)}{(k+1)(k+2) \cdots(k+n)} ; & & n=1,2, \ldots, k \\
& =0 ; & & n>k
\end{aligned}
$$

is a subordinating factor sequence for $k=1,2,3, \ldots$

These two theorems provide us with an excellent source of functions, namely the polynomials $V_{n}(z ; f)$, which are convex and are also subordinate to a given function $f(z) \in(K)$. Such sources are rare. Part II of Theorem 5 of this paper represents a slight contribution in the search for such functions.

The following conjecture appears in [6]:

CONJECtURe $C_{1}$. If $f(z)=\sum_{1}^{\infty} a_{n} z^{n}$ and $g(z)=\sum_{1}^{\infty} c_{n} z^{n}$ are regular in $|z|<1$ and map $|z|$ onto schlicht convex domains then so does the convolution $h(z)=f * g$ $=\sum_{1}^{\infty} a_{n} c_{n} z^{n}$.

Thus, for $f(z) \in(K)$, the functions $F_{p}(z)$ of Theorem 5 represent examples where the convolution of convex functions is convex and so is in agreement with Conjecture $C_{1}$. It has recently been announced by Suffridge [9] that the convolution of convex functions is close-to-convex.

A conjecture of Wilf [11] whose truth would imply Conjecture $C_{1}$ is

CONJECTURE $\mathrm{C}_{2}$. The coefficients of a convex function preserve subordination between convex functions. That is, if $\sum a_{n} z^{n}, \sum c_{n} z^{n}, \sum d_{n} z^{n}$ are regular and map $|z|<1$ onto schlicht convex domains, and if $\sum a_{n} z^{n} \prec \sum c_{n} z^{n}$ then $\sum a_{n} d_{n} z^{n} \prec \sum c_{n} d_{n} z^{n}$.

Some of the results in $\$ 6$ of this paper seem to support the truth of the following conjecture which I have not been able to prove. 
CONJeCture $C_{3}$. Let $f(z)=z+\sum_{2}^{\infty} a_{n} z^{n} \in(K), g(z)=\sum_{1}^{\infty} c_{n} z^{n}$ be regular and map $|z|<1$ univalently onto a convex domain, $c_{1}>0, g(z) \prec f(z)$ in $|z|<1, g(z) \not \equiv f(z)$. Then

$$
\begin{array}{ll}
\operatorname{Re} \frac{f(z)}{g(z)}>1 & \text { for }|z|<1, \\
\operatorname{Re} \frac{z f^{\prime}(z)}{f(z)-g(z)}>\frac{1}{2} & \text { for }|z|<1 .
\end{array}
$$

Note that in (a) the choice $g(z)=z / 2$ and in (b) the choice $g(z) \rightarrow 0$ yield, respectively, Strohhäcker's [8] sharp results $\operatorname{Re}(f(z) / z)>1 / 2, \operatorname{Re}\left(z f^{\prime}(z) / f(z)\right)>1 / 2$ for members $f(z)$ of the class $(K)$. In (a) the choice $f(z)=z /(1-z)$ and $g(z)=z / 2$ yields the result $\operatorname{Re}(1+z) /(1-z)>0$. Also, in view of Theorems $\mathrm{A}, \mathrm{B}$ the choice $g(z)=V_{n}(z ; f)$ in Conjecture $\mathrm{C}_{3}$ gives, for $|z|<1$,

$$
\operatorname{Re} \frac{f(z)}{V_{n}(z ; f)}>1 ; \quad \operatorname{Re} \frac{z f^{\prime}(z)}{f(z)-V_{n}(z ; f)}>\frac{1}{2} .
$$

Finally, we suggest the investigation of functions $F(z)$ defined by

$$
F(z)=\int_{0}^{1} f(t z) W(t) d t
$$

For what classes of weight functions $W(t)$, other than that given by (5.2) of Theorem 6 , will a property $T$ possessed by $f(z)$ (such as starlikeness, convexity, close-toconvexity) also be possessed by $F(z)$ ? (Note: The following counter example to Conjecture $\mathrm{C}_{3}$ has been communicated to me by T. J. Suffridge; $f(z)=z /(1-z)$, $g(z)=(2 / \pi) \arctan z$.)

\section{REFERENCES}

1. S. D. Bernardi, A survey of the development of the theory of schlicht functions, Duke Math. J. 19 (1952), 263-287.

2. - Special classes of subordinate functions, Duke Math. J. 33 (1966), 55-68.

3. W. Kaplan, Close-to-convex schlicht functions, Michigan Math. J. 1 (1952), 169-185.

4. R. J. Libera, Some classes of regular univalent functions. Proc. Amer. Math. Soc. 16 (1965), 755-758.

5. A. E. Livingston, On the radius of univalence of certain analytic functions, Proc. Amer. Math. Soc. 17 (1966), 352-357.

6. G. Pólya and I. J. Schoenberg, Remarks on de la Vallée Poussin means and convex conformal maps of the circle, Pacific J. Math. 8 (1958), 295-334.

7. M. S. Robertson, Application of the subordination principle to univalent functions, Pacific J. Math. 11 (1961), 315-324.

8. E. Strohhäcker, Beiträge zur Theorie der schlichten Funktionen, Math. Z. 37 (1933), 356380.

9. T. J. Suffridge, Convolutions of convex functions, Abstract 65T-149, Notices Amer. Math. Soc. 12 (1965), 244.

10. D. V. Widder, Laplace transforms, Princeton Univ. Press, Princeton, N. J., 1946.

11. H. S. Wilf, Subordinating factor sequences for convex maps of the unit circle, Proc. Amer. Math. Soc. 12 (1961), 689-693.

\section{NeW York UnIVERSITY,} BronX, NEW YorK 